\title{
A NEW NON-SYMMETRIC INFORMATION DIVERGENCE OF CSISZAR'S CLASS, PROPERTIES AND ITS BOUNDS
}

\author{
K.C.Jain ${ }^{1}$, Praphull Chhabra ${ }^{2}$ \\ ${ }^{1}$ Professor, Department of Mathematics, Malaviya National Institute of Technology, Jaipur (Rajasthan), India \\ ${ }^{2}$ Ph.d Scholar, Department of Mathematics, Malaviya National Institute of Technology, Jaipur (Rajasthan), India
}

\begin{abstract}
Non-parametric measures give the amount of information supplied by the data for discriminating in favor of a probability distribution $P$ against another $Q$, or for measuring the distance or affinity between $P$ and $Q$.

There are several generalized functional divergences, such as: Csiszar divergence, Renyi- like divergence, Bregman divergence, Burbea-Rao divergence etc. all. In this paper, a non-parametric non symmetric measure of divergence which belongs to the family of Csiszár's f-divergence is proposed. Its properties are studied and get the bounds in terms of some well known divergence measures.
\end{abstract}

Mathematics Subject Classification: 94A17, 26D15

Keywords: Csiszar's $f$ divergence, convex and normalized function, non-symmetric divergence measure, information inequalities, bounds of divergence measure.

\section{INTRODUCTION}

Let $\Gamma_{n}=\left\{P=\left(p_{1}, p_{2}, p_{3} \ldots, p_{n}\right): p_{i}>0, \sum_{i=1}^{n} p_{i}=1\right\}, n \geq 2$ be the set of all complete finite discrete probability distributions. If we take $p_{i} \geq 0$ for some $i=1,2,3, \ldots, n$, then we have to suppose that $0 f(0)=0 f\left(\frac{0}{0}\right)=0$.

Csiszar [2], given the generalized f- divergence measure, which is given by:

$C_{f}(P, Q)=\sum_{i=1}^{n} q_{i} f\left(\frac{p_{i}}{q_{i}}\right)$

Where $f:(0, \infty) \rightarrow R$ (set of real no.) is real, continuous and convex function and $P=\left(p_{1}, p_{2}, p_{3} \ldots, p_{n}\right), Q=\left(q_{1}, q_{2}, q_{3} \ldots, q_{n}\right) \in \Gamma_{\mathrm{n}}$, where $p_{i}$ and $q_{i}$ are probability mass functions. Many known divergences can be obtained from this generalized measure by suitably defining the convex function $\mathrm{f}$. Some of those are as follows:

- $\quad \chi^{2}(P, Q)=\sum_{i=1}^{n} \frac{\left(p_{i}-q_{i}\right)^{2}}{q_{i}}=$ Chi- Square divergence measure [5]

- $h(P, Q)=\sum_{i=1}^{n} \frac{\left(\sqrt{p_{i}}-\sqrt{q_{i}}\right)^{2}}{2}=$ Hellinger discrimination [3]

*

* $R_{a}(P, Q)=\sum_{i=1}^{n} \frac{p_{i}^{a}}{q_{i}^{a-1}}, a>1=$ Renyi's "a"

order entropy [6]

$*$

* $B(P, Q)=\sum_{i=1}^{n} \sqrt{p_{i} q_{i}} \quad=\quad$ Bhattacharya

divergence measure [1]

*

Relative information of type "s" [9]

* $\Phi_{s}(P, Q)=[s(s-1)]^{-1}\left[\sum_{i=1}^{n} p_{i}^{s} q_{i}^{1-s}-1\right], s \neq 0,1$ and $s \in R$

* $\quad K(P, Q)=\sum_{i=1}^{n} p_{i} \log \left(\frac{p_{i}}{q_{i}}\right)=$ Kullback- Leibler

divergence measure [4] 
Particularly

$\lim _{s \rightarrow 1} \Phi_{s}(P, Q)=K(P, Q), \lim _{s \rightarrow 0} \Phi_{s}(P, Q)=K(Q, P)$

Where $K(P, Q)$ is given by (1.2).

* $\quad G(P, Q)=\sum_{i=1}^{n}\left(\frac{p_{i}+q_{i}}{2}\right) \log \left(\frac{p_{i}+q_{i}}{2 p_{i}}\right)=$

Relative AG Divergence [7]

Similarly, we get many others divergences as well by defining suitable convex function

\section{NEW INFORMATION DIVERGENCE}

\section{MEASURE}

In this section, we shall obtain a new divergence measure corresponding to new convex function, and will study the properties.

The following theorem is well known in literature [2].

Theorem 1: If the function $\mathrm{f}$ is convex and normalized, i.e., $f(1)=0$, then $C_{f}(P, Q)$ and its ad joint $C_{f}(Q, P)$ are both non-negative and convex in the pair of probability distribution $(P, Q) \in \Gamma_{n} \times \Gamma_{n}$.

Let $f:(0, \infty) \rightarrow R$, be a mapping, defined as:

$$
f(t)=\frac{(t-1)^{4}}{t}, t \in(0, \infty)
$$

And

$f^{\prime}(t)=\frac{(t-1)^{3}}{t^{2}}(3 t+1), f^{\prime \prime}(t)=\frac{2(t-1)^{2}}{t^{3}}\left(3 t^{2}+2 t+1\right)$

Properties of function defined by (2.1), are as follows:

* Since $f^{\prime \prime}(t) \geq 0 \forall t \in(0, \infty) \Rightarrow f(t)$ is a convex function.

* Since $f(1)=0 \Rightarrow f(t)$ is a normalized function.

* Since $f^{\prime}(t)<0$ at $(0,1)$ and $f^{\prime}(t)>0$ at $(1, \infty) \Rightarrow f(t)$ is monotonically decreasing in $(0,1)$ and monotonically increasing in $(1, \infty)$, and $f^{\prime}(1)=0$.

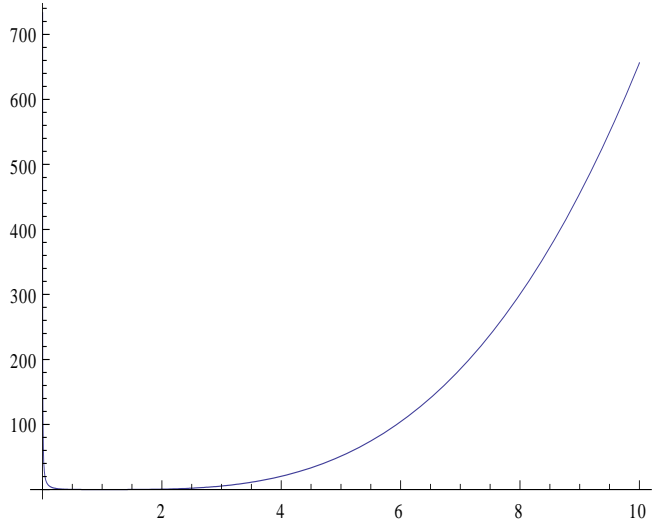

Fig1: Graph of convex function $f(t)$

Now, put (2.1) in (1.1), we get the following new divergence:

$$
C_{f}(P, Q)=V^{*}(P, Q)=\sum_{i=1}^{n} \frac{\left(p_{i}-q_{i}\right)^{4}}{p_{i} q_{i}^{2}}
$$

Properties of divergence defined by (2.3), are as follows:

* In view of theorem 1, we can say that $V^{*}(P, Q)>0$ and convex in the pair of probability distribution $(P, Q) \in \Gamma_{n} \times \Gamma_{n}$.

* $\quad V^{*}(P, Q)=0$ if $P=Q$ or $p_{i}=q_{i}$ (Attains its minimum value).

* Since $V^{*}(P, Q) \neq V^{*}(Q, P) \Rightarrow V^{*}(P, Q)$ is nonsymmetric divergence measure w.r.t. $P \& Q$.

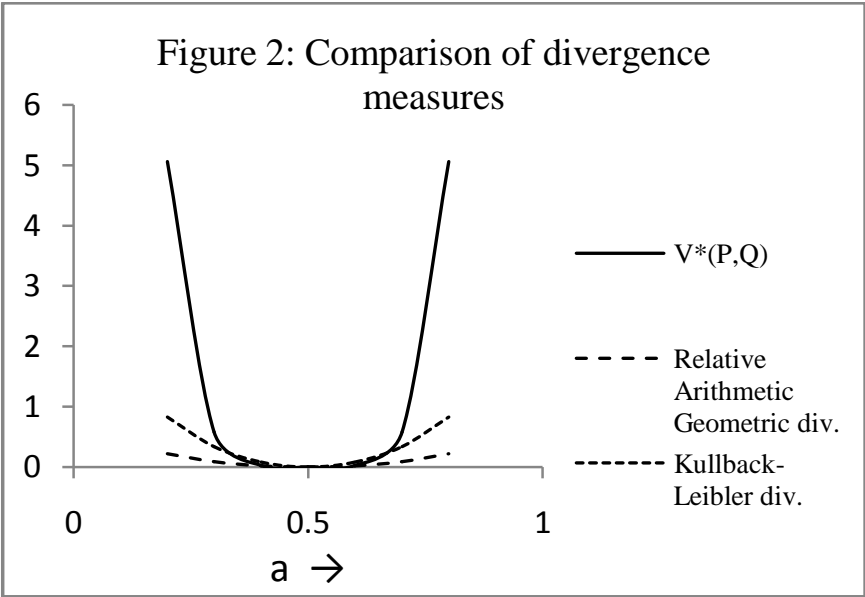

Figure 2 shows the behavior of $V *(P, Q)$, Relative Arithmetic-Geometric divergence $G(P, Q)$ and KullbackLeibler divergence $K(P, Q)$. We have considered $p_{i}=(a, 1-a)$ and $q_{i}=(1-a, a)$ where $a \in(0,1)$. It 
is clear from figure 2 that the new measure $V^{*}(P, Q)$ has a steeper slope then $G(P, Q)$ and $K(P, Q)$.

\section{CSISZAR'S FUNCTIONAL DIVERGENCE AND INEQUALITIES}

The following theorem is well known in literature [8].

Theorem 2: Let $f: I \subset R_{+} \rightarrow R$ (I is an open interval) be a mapping which is normalized, i.e., $f(1)=0$ and suppose that

I. $f$ is twice differentiable on $(\alpha, \beta), 0<\alpha \leq 1 \leq \beta<\infty$ with $\alpha \neq \beta$.

\subsection{Proposition 4.1 (at $s=2)$}

Let $\chi^{2}(P, Q)$ and $V *(P, Q)$ be defined as in (1.3) and (2.3) respectively. Then, we have

i. If $0<\alpha<1$, then

$0 \leq V^{*}(P, Q) \leq \max .\left\{\frac{(\alpha-1)^{2}}{\alpha^{3}}\left(3 \alpha^{2}+2 \alpha+1\right), \frac{(\beta-1)^{2}}{\beta^{3}}\left(3 \beta^{2}+2 \beta+1\right)\right\} \chi^{2}(P, Q)$

$0 \leq V_{\rho}^{*}(P, Q)-V^{*}(P, Q)$

$\leq \max .\left\{\frac{(\alpha-1)^{2}}{\alpha^{3}}\left(3 \alpha^{2}+2 \alpha+1\right), \frac{(\beta-1)^{2}}{\beta^{3}}\left(3 \beta^{2}+2 \beta+1\right)\right\} \chi^{2}(P, Q)$

ii. If $\alpha=1$, then

$0 \leq V^{*}(P, Q) \leq \frac{(\beta-1)^{2}}{\beta^{3}}\left(3 \beta^{2}+2 \beta+1\right) \chi^{2}(P, Q)$

$m<M$ and $m \leq t^{2-s} f^{\prime \prime}(t) \leq M \forall t \in(\alpha, \beta)$ and $s \in R$ and

$0 \leq V_{\rho}^{*}(P, Q)-V^{*}(P, Q) \leq \frac{(\beta-1)^{2}}{\beta^{3}}\left(3 \beta^{2}+2 \beta+1\right) \chi^{2}(P, Q)$

$m \Phi_{s}(P, Q) \leq C_{f}(P, Q) \leq M \Phi_{s}(P, Q)$

And

\section{Proof:}

Firstly, put s=2 in (1.7) and (3.4) respectively, we get

$m\left[\eta_{s}(P, Q)-\Phi_{s}(P, Q)\right] \leq C_{\rho}(P, Q)-C_{f}(P, Q) \leq M\left[\eta_{s}(P, Q)-\Phi_{s}(P, Q)\right]$

$\Phi_{s}(P, Q)=\frac{1}{2} \sum_{i=1}^{n} \frac{p_{i}^{2}}{q_{i}}-1=\frac{1}{2} \sum_{i=1}^{n} \frac{p_{i}^{2}}{q_{i}}-2 p_{i}+q_{i}=\frac{1}{2} \sum_{i=1}^{n} \frac{\left(p_{i}-q_{i}\right)^{2}}{q_{i}}=\frac{1}{2} \chi^{2}(P, Q)$

Where

$C_{\rho}(P, Q)=C_{f^{\prime}}\left(\frac{P^{2}}{Q}, P\right)-C_{f^{\prime}}(P, Q)=\sum_{i=1}^{n}\left(p_{i}-q_{i}\right) f^{\prime}\left(\frac{p_{i}}{q_{i}}\right)$

$\eta_{s}(P, Q)=C_{\Phi_{s}^{\prime}}\left(\frac{P^{2}}{Q}, P\right)-C_{\Phi_{s}^{\prime}}(P, Q)=(s-1)^{-1} \sum_{i=1}^{n}\left(p_{i}-q_{i}\right)\left(\frac{p_{i}}{q_{i}}\right)^{s-1}, s \neq 1$

And $C_{f}(P, Q), \Phi_{s}(P, Q)$ are given by (1.1) and (1.7) respectively

\section{BOUNDS OF NEW INFORMATION} DIVERGENCE MEASURE

In this section, we derive bounds for $V^{*}(P, Q)$ in terms of the well known divergences in the following propositions at $s=2,1,1 / 2,0$ and -1 , by using the theorem 2 .
$\eta_{s}(P, Q)=\sum_{i=1}^{n}\left(p_{i}-q_{i}\right) \frac{p_{i}}{q_{i}}=\sum_{i=1}^{n} \frac{p_{i}^{2}}{q_{i}}-p_{i}=\sum_{i=1}^{n} \frac{p_{i}^{2}}{q_{i}}-1$

$=\sum_{i=1}^{n} \frac{p_{i}^{2}}{q_{i}}-2 p_{i}+q_{i}=\sum_{i=1}^{n} \frac{\left(p_{i}-q_{i}\right)^{2}}{q_{i}}=\chi^{2}(P, Q)$

And by putting $f^{\prime}(t)$ in (3.3), we get

$V_{\rho}^{*}(P, Q)=V_{f^{\prime}}^{*}\left(\frac{P^{2}}{Q}, P\right)-V_{f^{\prime}}^{*}(P, Q)=\sum_{i=1}^{n} \frac{\left(p_{i}-q_{i}\right)^{4}}{\left(p_{i} q_{i}\right)^{2}}\left(3 p_{i}+q_{i}\right)$

Let $\quad g(t)=f^{\prime \prime}(t)=\frac{2(t-1)^{2}}{t^{3}}\left(3 t^{2}+2 t+1\right) \quad$ (After putting $\mathrm{s}=2$ in $\left.t^{2-s} f^{\prime \prime}(t)\right)$ 
Then $\quad g^{\prime}(t)=\frac{6\left(t^{4}-1\right)}{t^{4}}, g^{\prime \prime}(t)=\frac{24}{t^{5}}$

If $g^{\prime}(t)=0 \Rightarrow t^{4}-1=0 \Rightarrow t=1,-1$

It is clear that $\mathrm{g}(\mathrm{t})$ is monotonic decreasing on $(0,1)$ and monotonic increasing on $[1, \infty)$.

Also $g$ (t) has minimum value at $t=1$, since $g^{\prime \prime}(1)=24>0$ so

$m=\inf _{t \in(0, \infty)} g(t)=g(1)=0$

Now, we have two cases:

i. If $0<\alpha<1$, then

$$
\begin{aligned}
& M=\sup _{t \in(\alpha, \beta)} g(t)=\max \{g(\alpha), g(\beta)\} \\
& =\max \left\{\frac{2(\alpha-1)^{2}}{\alpha^{3}}\left(3 \alpha^{2}+2 \alpha+1\right), \frac{2(\beta-1)^{2}}{\beta^{3}}\left(3 \beta^{2}+2 \beta+1\right)\right\}
\end{aligned}
$$

ii. If $\alpha=1$, then

$$
M=\sup _{t \in[1, \beta)} g(t)=\frac{2(\beta-1)^{2}}{\beta^{3}}\left(3 \beta^{2}+2 \beta+1\right)
$$

The results (4.1), (4.2), (4.3) and (4.4) are obtained by using (2.3), (4.5), (4.6), (4.7), (4.8), (4.9) and (4.10) in 3.1 and 3.2.

\subsection{Proposition $4.2($ at $s=1)$}

Let $K(P, Q)$ and $V *(P, Q)$ be defined as in (1.2) and (2.3) respectively. Then, we have

i. If $0<\alpha<1$, then

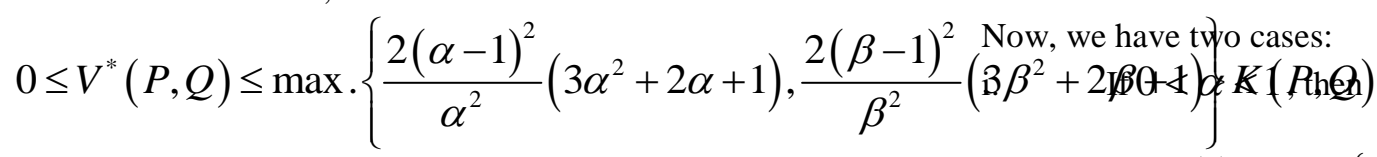

$0 \leq V_{\rho}^{*}(P, Q)-V^{*}(P, Q)$

$\leq \max .\left\{\frac{2(\alpha-1)^{2}}{\alpha^{2}}\left(3 \alpha^{2}+2 \alpha+1\right), \frac{2(\beta-1)^{2}}{\beta^{2}}\left(3 \beta^{2}+2 \beta+1\right)\right\} K(Q, P)$

ii. If $\alpha=1$, then

$0 \leq V^{*}(P, Q) \leq \frac{2(\beta-1)^{2}}{\beta^{2}}\left(3 \beta^{2}+2 \beta+1\right) K(P, Q)$
$0 \leq V_{\rho}^{*}(P, Q)-V^{*}(P, Q) \leq \frac{2(\beta-1)^{2}}{\beta^{2}}\left(3 \beta^{2}+2 \beta+1\right) K(Q, P)$

Proof:

Firstly, put s=1 in (1.7) and (3.4) respectively, we get

$$
\lim _{s \rightarrow 1} \Phi_{s}(P, Q)=\sum_{i=1}^{n} p_{i} \log \left(\frac{p_{i}}{q_{i}}\right)=K(P, Q)
$$

$\lim _{s \rightarrow 1} \eta_{s}(P, Q)=\sum_{i=1}^{n} p_{i} \log \left(\frac{p_{i}}{q_{i}}\right)+q_{i} \log \left(\frac{q_{i}}{p_{i}}\right)=K(P, Q)+K(Q, P)$

Let $\quad g(t)=t f^{\prime \prime}(t)=\frac{2(t-1)^{2}}{t^{2}}\left(3 t^{2}+2 t+1\right) \quad$ (After putting $s=1$ in $\left.t^{2-s} f^{\prime \prime}(t)\right)$

Then

$g^{\prime}(t)=\frac{4(t-1)}{t^{3}}\left(3 t^{3}+t^{2}+t+1\right), g^{\prime \prime}(t)=\frac{12\left(t^{4}+1\right)}{t^{4}}$

If

$$
g^{\prime}(t)=0 \Rightarrow(t-1)\left(3 t^{3}+t^{2}+t+1\right)=0 \Rightarrow t=1,-0.63
$$

It is clear that $\mathrm{g}(\mathrm{t})$ is monotonic decreasing on $(0,1)$ and monotonic increasing on $[1, \infty)$.

Also $g$ (t) has minimum value at $\mathrm{t}=1$, since $g^{\prime \prime}(1)=24>0$ so

$m=\inf _{t \in(0, \infty)} g(t)=g(1)=0$

$$
\begin{aligned}
& M=\sup _{t \in(\alpha, \beta)} g(t)=\max \{g(\alpha), g(\beta)\} \\
& =\max \left\{\frac{2(\alpha-1)^{2}}{\alpha^{2}}\left(3 \alpha^{2}+2 \alpha+1\right), \frac{2(\beta-1)^{2}}{\beta^{2}}\left(3 \beta^{2}+2 \beta+1\right)\right\}
\end{aligned}
$$

ii. If $\alpha=1$, then

$$
M=\sup _{t \in[1, \beta)} g(t)=\frac{2(\beta-1)^{2}}{\beta^{2}}\left(3 \beta^{2}+2 \beta+1\right)
$$


The results (4.11), (4.12), (4.13) and (4.14) are obtained by using (2.3), (4.7), (4.15), (4.16), (4.17), (4.18), and (4.19) in 3.1 and 3.2 .

\subsection{Proposition 4.3(at $s=1 / 2)$}

Let $h(P, Q), \mathrm{R}_{a}(P, Q), \mathrm{B}(P, Q)$ and $V^{*}(P, Q)$ be defined as in (1.4), (1.5), (1.6) and (2.3) respectively. Then, we have

i. If $0<\alpha<1$, then

$0 \leq V^{*}(P, Q) \leq \max \left\{\frac{8(\alpha-1)^{2}}{\alpha^{3 / 2}}\left(3 \alpha^{2}+2 \alpha+1\right), \frac{8(\beta-1)^{2}}{\beta^{3 / 2}}\left(3 \beta^{2}+2 \beta+1\right)\right\} h(P, Q)$

$0 \leq V_{\rho}^{*}(P, Q)-V^{*}(P, Q)$

$\leq \max \left\{\frac{8(\alpha-1)^{2}}{\alpha^{3 / 2}}\left(3 \alpha^{2}+2 \alpha+1\right), \frac{8(\beta-1)^{2}}{\beta^{3 / 2}}\left(3 \beta^{2}+2 \beta+1\right)\right\}\left[\frac{1}{2}\left\{R_{3 / 2}(Q, P)-B(P, Q)\right\}-h(P, Q)\right]$

ii. If $\alpha=1$, then

$0 \leq V^{*}(P, Q) \leq \frac{8(\beta-1)^{2}}{\beta^{3 / 2}}\left(3 \beta^{2}+2 \beta+1\right) h(P, Q)$

$0 \leq V_{\rho}^{*}(P, Q)-V^{*}(P, Q)$

$\leq \frac{8(\beta-1)^{2}}{\beta^{3 / 2}}\left(3 \beta^{2}+2 \beta+1\right)\left[\frac{1}{2}\left\{R_{3 / 2}(Q, P)-B(P, Q)\right\}-h(P, Q)\right]$

Proof:

Firstly, put s=1/2 in (1.7) and (3.4) respectively, we get

$\Phi_{s}(P, Q)=4 \sum_{i=1}^{n} 1-\sqrt{p_{i} q_{i}}=2 \sum_{i=1}^{n} 2-2 \sqrt{p_{i} q_{i}}$

$=2 \sum_{i=1}^{n} p_{i}+q_{i}-2 \sqrt{p_{i} q_{i}}=4 \sum_{i=1}^{n} \frac{\left(\sqrt{p_{i}}-\sqrt{q_{i}}\right)^{2}}{2}=4 h(P, Q)$

$\eta_{s}(P, Q)=2 \sum_{i=1}^{n}\left(q_{i}-p_{i}\right) \sqrt{\frac{q_{i}}{p_{i}}}=2 \sum_{i=1}^{n}\left(\frac{q_{i}^{3 / 2}}{p_{i}^{1 / 2}}-\sqrt{p_{i} q_{i}}\right)=2\left[R_{3 / 2}(Q, P)-B(P, Q)\right]$ (4.25)
Let $\quad g(t)=t^{\frac{3}{2}} f^{\prime \prime}(t)=\frac{2(t-1)^{2}}{t^{\frac{3}{2}}}\left(3 t^{2}+2 t+1\right)$

(After

putting $\mathrm{s}=1 / 2$ in $\left.t^{2-s} f^{\prime \prime}(t)\right)$

Then $g^{\prime}(t)=\frac{3(t-1)}{t^{\frac{5}{2}}}\left(5 t^{3}+t^{2}+t+1\right), g^{\prime \prime}(t)=\frac{3}{2 t^{\frac{7}{2}}}\left(15 t^{4}-4 t^{3}+5\right)$

If $g^{\prime}(t)=0 \Rightarrow(t-1)\left(5 t^{3}+t^{2}+t+1\right)=0 \Rightarrow t=1,-0.53$

It is clear that $\mathrm{g}(\mathrm{t})$ is monotonic decreasing on $(0,1)$ and monotonic increasing on $[1, \infty)$.

Also $g$ (t) has minimum value at $\mathrm{t}=1$, since $g^{\prime \prime}(1)=24>0$ so

$m=\inf _{t \in(0, \infty)} g(t)=g(1)=0$

Now, we have two cases:

i. If $0<\alpha<1$, then

$$
\begin{aligned}
& M=\sup _{t \in(\alpha, \beta)} g(t)=\max \{g(\alpha), g(\beta)\} \\
& =\max \left\{\frac{2(\alpha-1)^{2}}{\alpha^{\frac{3}{2}}}\left(3 \alpha^{2}+2 \alpha+1\right), \frac{2(\beta-1)^{2}}{\beta^{\frac{3}{2}}}\left(3 \beta^{2}+2 \beta+1\right)\right\}
\end{aligned}
$$

ii. If $\alpha=1$, then

$$
M=\sup _{t \in[1, \beta)} g(t)=\frac{2(\beta-1)^{2}}{\beta^{\frac{3}{2}}}\left(3 \beta^{2}+2 \beta+1\right)
$$

The results (4.20), (4.21), (4.22) and (4.23) are obtained by using (2.3), (4.7), (4.24), (4.25), (4.26), (4.27), and (4.28) in 3.1 and 3.2 .

\subsection{Proposition $4.4($ at $s=0)$}

Let $K(P, Q), \chi^{2}(P, Q)$ and $V *(P, Q)$ be defined as in (1.2), (1.3) and (2.3) respectively. Then, we have

i. If $0<\alpha<1$, then

$0 \leq V^{*}(P, Q) \leq \max \left\{\frac{2(\alpha-1)^{2}}{\alpha}\left(3 \alpha^{2}+2 \alpha+1\right), \frac{2(\beta-1)^{2}}{\beta}\left(3 \beta^{2}+2 \beta+1\right)\right\} K(Q, P)$ 
$0 \leq V_{\rho}^{*}(P, Q)-V^{*}(P, Q)$

$\leq \max \left\{\frac{2(\alpha-1)^{2}}{\alpha}\left(3 \alpha^{2}+2 \alpha+1\right), \frac{2(\beta-1)^{2}}{\beta}\left(3 \beta^{2}+2 \beta+1\right)\right\}\left\{\chi^{2}(Q, P)-K(Q, P)\right\}$

(4.30)

ii. If $\alpha=1$, then

$0 \leq V^{*}(P, Q) \leq \frac{2(\beta-1)^{2}}{\beta}\left(3 \beta^{2}+2 \beta+1\right) K(Q, P)$

$0 \leq V_{\rho}^{*}(P, Q)-V^{*}(P, Q) \leq \frac{2(\beta-1)^{2}}{\beta}\left(3 \beta^{2}+2 \beta+1\right)\left\{\chi^{2}(Q, P)-K(Q, P)\right\}$

\section{Proof:}

Firstly, put s=0 in (1.7) and (3.4) respectively, we get

$\lim _{s \rightarrow 0} \Phi_{s}(P, Q)=\sum_{i=1}^{n} q_{i} \log \left(\frac{q_{i}}{p_{i}}\right)=K(Q, P)$

$\eta_{s}(P, Q)=\sum_{i=1}^{n} \frac{q_{i}^{2}}{p_{i}}-q_{i}=\sum_{i=1}^{n} \sum_{i}^{2}-1=\sum_{i=1}^{n} \frac{q_{i}^{2}}{p_{i}}-2 q_{i}+p_{i}=\sum_{i=1}^{n} \frac{\left(p_{i}-q_{i}\right)^{2}}{p_{i}}=\chi^{2}(Q, P)$ (4.34)

Let $g(t)=t^{2} f^{\prime \prime}(t)=\frac{2(t-1)^{2}}{t}\left(3 t^{2}+2 t+1\right) \quad$ (After putting $\mathrm{s}=0$ in $\left.t^{2-s} f^{\prime \prime}(t)\right)$

Then

$$
\begin{aligned}
& g^{\prime}(t)=\frac{2(t-1)}{t^{2}}\left(9 t^{3}+t^{2}+t+1\right), g^{\prime \prime}(t)=\frac{1}{t^{3}}\left(36 t^{4}-16 t^{3}+\right. \\
& \text { If } \\
& g^{\prime}(t)=0 \Rightarrow(t-1)\left(9 t^{3}+t^{2}+t+1\right)=0 \Rightarrow t=1,-0.43
\end{aligned}
$$

It is clear that $\mathrm{g}(\mathrm{t})$ is monotonic decreasing on $(0,1)$ and monotonic increasing on $[1, \infty)$.

Also $g$ (t) has minimum value at $\mathrm{t}=1$, since $g^{\prime \prime}(1)=24>0$ so
Now, we have two cases:

i. If $0<\alpha<1$, then

$$
\begin{aligned}
& M=\sup _{t \in(\alpha, \beta)} g(t)=\max \{g(\alpha), g(\beta)\} \\
& =\max \left\{\frac{2(\alpha-1)^{2}}{\alpha}\left(3 \alpha^{2}+2 \alpha+1\right), \frac{2(\beta-1)^{2}}{\beta}\left(3 \beta^{2}+2 \beta+1\right)\right\}
\end{aligned}
$$

ii. If $\alpha=1$, then

$$
M=\sup _{t \in[1, \beta)} g(t)=\frac{2(\beta-1)^{2}}{\beta}\left(3 \beta^{2}+2 \beta+1\right)
$$

The results (4.29), (4.30), (4.31) and (4.32) are obtained by using (2.3), (4.7), (4.33), (4.34), (4.35), (4.36), and (4.37) in 3.1 and 3.2 .

\subsection{Proposition 4.5 (at $s=-1)$}

Let $\chi^{2}(P, Q), \mathrm{R}_{a}(P, Q)$ and $V *(P, Q)$ be defined as in (1.3), (1.5) and (2.3) respectively. Then, we have

i. If $0<\alpha<1$, then

$0 \leq V^{*}(P, Q) \leq \max \left\{(\alpha-1)^{2}\left(3 \alpha^{2}+2 \alpha+1\right),(\beta-1)^{2}\left(3 \beta^{2}+2 \beta+1\right)\right\} \chi^{2}(Q, P)$

$0 \leq V_{\rho}^{*}(P, Q)-V^{*}(P, Q)$

$\leq \max \left\{(\alpha-1)^{2}\left(3 \alpha^{2}+2 \alpha+1\right),(\beta-1)^{2}\left(3 \beta^{2}+2 \beta+1\right)\right)\left\{R_{2}(Q, P)-R_{3}(Q, P)-\chi^{2}(Q, P)\right\}$

$$
m=\inf _{t \in(0, \infty)} g(t)=g(1)=0
$$$$
0 \leq V^{*}(P, Q) \leq(\beta-1)^{2}\left(3 \beta^{2}+2 \beta+1\right) \chi^{2}(Q, P)
$$

$0 \leq V_{\rho}^{*}(P, Q)-V^{*}(P, Q) \leq(\beta-1)^{2}\left(3 \beta^{2}+2 \beta+1\right)\left\{R_{3}(Q, P)-R_{2}(Q, P)-\chi^{2}(Q, P)\right\}$

Proof:

Firstly, put s=-1 in (1.7) and (3.4) respectively, we get $\Phi_{s}(P, Q)=\frac{1}{2} \sum_{i=1}^{n} \frac{q_{i}^{2}}{p_{i}}-1=\frac{1}{2} \sum_{i=1}^{n} \frac{q_{i}^{2}}{p_{i}}-2 q_{i}+p_{i}=\frac{1}{2} \sum_{i=1}^{n} \frac{\left(p_{i}-q_{i}\right)^{2}}{p_{i}}=\frac{1}{2} \chi^{2}(Q, P)$ 
$\eta_{s}(P, Q)=\frac{1}{2} \sum_{i=1}^{n}\left(q_{i}-p_{i}\right) \frac{q_{i}^{2}}{p_{i}^{2}}=\frac{1}{2} \sum_{i=1}^{n}\left(\frac{q_{i}^{3}}{p_{i}^{2}}-\frac{q_{i}^{2}}{p_{i}}\right)=\frac{1}{2}\left[R_{3}(Q, P)-R_{2}(Q, P)\right]$

Let $g(t)=t^{3} f^{\prime \prime}(t)=2(t-1)^{2}\left(3 t^{2}+2 t+1\right) \quad$ (After putting $\mathrm{s}=-1$ in $\left.t^{2-s} f^{\prime \prime}(t)\right)$

Then $g^{\prime}(t)=24 t^{2}(t-1), g^{\prime \prime}(t)=72 t^{2}-48 t$

If $g^{\prime}(t)=0 \Rightarrow t=0,1$

It is clear that $\mathrm{g}(\mathrm{t})$ is monotonic decreasing on $(0,1)$ and monotonic increasing on $[1, \infty)$.

Also $g$ (t) has minimum value at $\mathrm{t}=1$, since $g^{\prime \prime}(1)=24>0$ so

$m=\inf _{t \in(0, \infty)} g(t)=g(1)=0$

Now, we have two cases:

i. If $0<\alpha<1$, then

$$
\begin{aligned}
& M=\sup _{t \in(\alpha, \beta)} g(t)=\max \{g(\alpha), g(\beta)\} \\
& =\max \left\{2(\alpha-1)^{2}\left(3 \alpha^{2}+2 \alpha+1\right), 2(\beta-1)^{2}\left(3 \beta^{2}+2 \beta+1\right)\right\}
\end{aligned}
$$

ii. If $\alpha=1$, then

$$
M=\sup _{t \in[1, \beta)} g(t)=2(\beta-1)^{2}\left(3 \beta^{2}+2 \beta+1\right)
$$

The results $(4.38),(4.39),(4.40)$ and $(4.41)$ are obtained by using (2.3), (4.7), (4.42), (4.43), (4.44), (4.45), and (4.46) in 3.1 and 3.2 .

\section{REFERENCES}

[1]. Bhattacharyya A., "On some analogues to the amount of information and their uses in statistical estimation", Sankhya, 8, 1-14.

[2]. Csiszar I., "Information type measures of differences of probability distribution and indirect observations", Studia Math. Hungarica, 2(1967), 299-318.

[3]. Hellinger E., "Neue begrundung der theorie der quadratischen formen von unendlichen vielen veranderlichen", J. Rein.Aug. Math., 136(1909), 210-271.

[4]. Kullback S. and Leibler R.A., "On Information and Sufficiency”, Ann. Math. Statist., 22(1951), 79-86.
[5]. Pearson K., "On the Criterion that a given system of deviations from the probable in the case of correlated system of variables is such that it can be reasonable supposed to have arisen from random sampling", Phil. Mag., 50(1900), 157-172.

[6]. Renyi A., "On measures of entropy and information", Proc. 4th Berkeley Symposium on Math. Statist. and Prob., 1(1961), 547-561.

[7]. Taneja I.J., New developments in generalized information measures, Chapter in: Advances in Imaging and Electron Physics, Ed. P.W. Hawkes, 91(1995), 37-135.

[8]. Taneja I. J. and Kumar P., "Generalized non-symmetric divergence measures and inequalities" (2000), The Natural Science and Engineering Research Council's Discovery grant to Pranesh Kumar.

[9]. Taneja I.J. and Kumar P., "Relative Information of type$\mathrm{s}$, Csiszar's $f$-divergence and information inequalities", Information Sciences, (2003). 